\title{
IKERLANAK
}

\section{QUATERNARY DICHOTOMOUS VOTING RULES}

by

Annick Laruelle and Federico Valenciano

2010

Working Paper Series: IL. 41/10

Departamento de Fundamentos del Análisis Económico I

Ekonomi Analisiaren Oinarriak I Saila

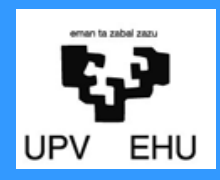

University of the Basque Country 


\title{
Quaternary dichotomous voting rules*
}

\author{
Annick Laruelletand Federico Valenciano ${ }^{\S}$
}

January 26, 2010

\begin{abstract}
In this paper we provide a general model of 'quaternary' dichotomous voting rules (QVRs), namely, voting rules for making collective dichotomous decisions (to accept or reject a proposal), based on vote profiles in which four options are available to each voter: voting ('yes', 'no' or 'abstaining') or staying home and not turning out. The model covers most of actual real-world dichotomus rules, where quorums are often required, and some of the extensions considered in the literature. In particular, we address and solve the question of the representability of QVRs by means of weighted rules and extend the notion of 'dimension' of a rule.

JEL Classification: C71, D71
\end{abstract}

\section{Introduction}

Most of the literature on dichotomous decision-making considers only binary voting rules, where the passage or rejection of a proposal is decided on the basis of the votes cast by those who vote 'yes'. This implicitly assumes either that voting 'yes' and 'not' are the only feasible options, or that abstention and not showing up are counted as 'noes'. In the real world rules often distinguish between these three options. A well-known example is the UN Security Council. A proposal can be passed if at least nine out of the fifteen members are in favor and no veto member is against (i.e. all veto members approve or abstain). In many Parliaments the requirement for passing

*This research is supported by the Spanish Ministerio de Ciencia e Innovación under projects SEJ2006-05455 and ECO2009-11213, co-funded by ERDF, and from the Basque Government's funding to Grupo Consolidado GIC07/146-IT-377-07. We thank Jorge Alcalde, Alaitz Artabe and Miguel A. Ballester for their comments.

†Departamento de Fundamentos del Análisis Económico I, Universidad del País Vasco, Avenida Lehendakari Aguirre, 83, E-48015 Bilbao, Spain; a.laruelle@ikerbasque.org.

${ }^{\ddagger}$ IKERBASQUE, Basque Foundation of Science, 48011, Bilbao, Spain.

$\S$ Departamento de Economía Aplicada IV, Universidad del País Vasco, Avenida Lehendakari Aguirre, 83, E-48015 Bilbao, Spain; federico.valenciano@ehu.es. 
a bill is often based on the number of MPs present, not on the total number of MPs. Other examples include all rules with participation quora, that is, those that require the presence of a minimum number of voters for a vote to take place.

Felsenthal and Machover (1997) introduce abstention as a third option and deal with the measurement of power in this context. Freixas and Zwicker (2003) study rules with any number of ordered options or levels of support in the input, and any number of ordered levels of approval in the output. They extend the notion of weighted majorities for these rules. Freixas and Zwicker (2009) and Zwicker (2009) focus on anonymous ordered voting rules. Côrte-Real and Pereira (2004) and Maniquet and Morelli (2008) study ternary rules where the three actions are voting 'yes', voting 'no', and not participating. They study the strategic aspect induced by the participation quorum. Dougherty and Edward (2010) compare the simple majority and the absolute majority in a context where all four options are possible.

In this paper we extensively study quaternary dichotomous rules. The four possible options are those mentioned above, and the outcome is dichotomous, i.e. either acceptance or rejection of a proposal. This paper is complementary to Freixas and Zwicker (2003, 2009) and Zwicker (2009). On the one hand, they consider $j$ options and $k$ outcomes, while we focus on 4 inputs and 2 outputs. On the other hand, we consider the case where levels of support are not necessarily ordered, while they do not. In particular we study rules with participation quora, where the 'not participating' option and the 'no' option cannot be ranked: depending on the vote profile, the 'no' option may be more favorable or less favorable to the rejection of the proposal than the "not participating' option. We extend their notion of weighted rules, and also define the notion of dimension in this context and prove its well definedness.

The paper is organized as follows. In Section 2 the general model of 'quaternary' dichotomous voting rule (QVR) is introduced. In Section 3 a lattice of 'monotonic' classes of QVRs (i.e. specified by an admissible notion of monotonicity) is introduced and its basic properties are studied. In Section 4 some special classes are described in some detail, their relationship with models or classes considered in the literature discussed, and examples from the real world are provided for some of them. In Section 5 the notions of weighted majority rule and dimension are extended for the class of rules introduced here, and the well-definedness of the notion of dimension is proved. Finally, we briefly summarize our conclusions and point out some lines of further research.

\section{Quaternary dichotomous rules}

A dichotomous voting rule specifies a collective decision, acceptance or rejection, for each possible vote profile. In the binary case usually considered in the literature (see for instance Laruelle and Valenciano, 2008) voters can only cast either a positive or a negative vote (or any action other than voting 'yes' counts as voting 'no').

If $n$ is the number of seats on the committee, we label them by $1,2, \ldots, n$, and $\mathcal{N}=\{1,2, \ldots, n\}$. The same labels are also used to designate the voters that occupy 
the corresponding seats. A (binary) vote configuration is a 2-partition of $\mathcal{N},\left(S^{Y}, S^{N}\right)$, where $S^{Y}$ is the set of yes voters. Then a binary dichotomous voting rule specifies a set $\mathcal{V} \subseteq 2^{\mathcal{N}}$ of 'winning configurations' (i.e. those which lead to the acceptance of the proposal)

$$
\mathcal{V}=\left\{S=\left(S^{Y}, S^{N}\right) \in 2^{\mathcal{N}}: S \text { leads to acceptance }\right\}
$$

that satisfy the following conditions. First, if all voters vote 'yes', the proposal should be adopted ('full-support' condition): $(\mathcal{N}, \varnothing) \in \mathcal{V}$; second, if all voters vote 'no' (or none votes 'yes') the proposal should be rejected ('null-support' condition): $(\varnothing, \mathcal{N}) \notin$ $\mathcal{V}$; and third, if a vote configuration is winning, then any other configuration with a larger set of 'yes'-voters is also winning ('monotonicity' condition): if $S \in \mathcal{V}$ and $S^{Y} \subseteq T^{Y}$ then $T \in \mathcal{V}$. A fourth condition is usually added. The possibility of a proposal and its negation both being accepted should be prevented. If a proposal leads to a configuration $S$, its opposite should lead to the configuration $T$ where $T^{Y}=S^{N}$. Both configurations should not be winning: if $S \in \mathcal{V}$ then $T \notin \mathcal{V}$. In this case the rule is called proper. A binary dichotomous rule that does not satisfy this condition is said to be improper. Most binary rules in the real world are proper. Nevertheless, it proves convenient to widen the class so as to include improper rules within the domain of voting rules.

In general, more options than 'yes' or 'no' are offered to voters and the final outcome may be sensitive to these options. Here we are interested in this more general type of voting rule. We consider the quaternary case where four different options are offered to each voter: a voter may not show up at the vote, may turn up but abstain, may come and vote yes or may come and vote no ${ }^{1}$. The precise account of a particular vote is specified by a vote configuration or vote profile $S=\left(S^{Y}, S^{A}, S^{H}, S^{N}\right)$ that keeps track of the action taken by the voter occupying each seat, where $S^{Y}$ is the set of 'yes'-voters, $S^{N}$ is the set of 'no'-voters, $S^{A}$ is the set of those who abstain and $S^{H}$ that of those who stay at home. A (quaternary) vote configuration is thus in general a 4-partition of $\mathcal{N}$, i.e. any two of these subsets are disjoint and $\mathcal{N}=S^{Y} \cup S^{A} \cup S^{N} \cup S^{H}$. The number of 'yes'-voters in the configuration $S$ is denoted by $s^{Y}$, and $s^{N}$ denotes the number of 'no'-voters, etc. We denote by $4^{\mathcal{N}}$ the $\operatorname{set}^{2}$ of 4 -partitions of $\mathcal{N}$.

A quaternary dichotomous voting rule based on this input should specify a set $\mathcal{W} \subseteq 4^{\mathcal{N}}$ of 'winning configurations'3

$$
\mathcal{W}=\left\{S=\left(S^{Y}, S^{A}, S^{H}, S^{N}\right) \in 4^{\mathcal{N}}: S \text { leads to acceptance }\right\}
$$

\footnotetext{
${ }^{1}$ In fact generality can be pushed a bit further by including the possibility of spoilt votes as a separate option, but they are most often identified with abstention or absence.

${ }^{2}$ This is an abbreviated notation for the set of maps from $\mathcal{N}$ to the set of actions $\{Y, A, H, N\}$, whose usual set-theoretic notation is $\{Y, A, H, N\}^{\mathcal{N}}$.

${ }^{3}$ A more suitable term in this setting, where a vote profile cannot be summarized by the set of 'yes'voters as it must include at least those that have chosen three different actions, would be 'accepting configuration' or 'yes-winning configuration'. Nevertheless, for the sake of simplicity we maintain the term 'winning configuration'.
} 
Now let us consider what conditions may reasonably be imposed on such a set of winning configurations for a sound and general enough notion of voting rule. Note that the monotonicity as formulated for binary dichotomous rules does not hold in general for actual rules with a quorum. Now a configuration can be winning, while a configuration with a larger set of 'yes'-voters (and a smaller set of 'no'-voters) may be losing, as the following example shows:

Example 1 In the Belgian Parliament a bill must receive more votes in favor than votes against in order to be passed, and a participation quorum of 76 is required. If 50 MPs go and vote 'yes', 30 go and vote 'no' and 70 are absent, the proposal is accepted, while if 60 MPs vote 'yes' and 90 are absent, the proposal is rejected.

In the example the set of 'yes'-voters is not extended exclusively at the expense of the set of no-voters, the set of voters who stay at home also becomes larger. If the set of 'yes'-voters is extended exclusively at the expense of the 'no'-voters a winning configuration should not become losing. We refer to this condition as NY-monotonicity. In similar terms we define and assume AY-monotonicity (extension of the set of 'yes'voters solely at the expense of the abstainers), and HY-monotonicity (extension of the set of 'yes'-voters solely at the expense of the voters who stay at home). We thus have three monotonicity conditions:

$\boldsymbol{N} \boldsymbol{Y}$-monotonicity: If $S \in \mathcal{W}$, then $T \in \mathcal{W}$ for any $T$ such that $S^{Y} \subseteq T^{Y}, S^{A}=T^{A}$ and $S^{H}=T^{H}$.

$\boldsymbol{A} \boldsymbol{Y}$-monotonicity: If $S \in \mathcal{W}$, then $T \in \mathcal{W}$ for any $T$ such that $S^{Y} \subseteq T^{Y}, S^{N}=T^{N}$ and $S^{H}=T^{H}$.

$\boldsymbol{H} \boldsymbol{Y}$-monotonicity: If $S \in \mathcal{W}$, then $T \in \mathcal{W}$ for any $T$ such that $S^{Y} \subseteq T^{Y}, S^{A}=T^{A}$ and $S^{N}=T^{N}$.

The following diagram represents these three monotonicities, that is, the transitions of votes that keep a winning vote configuration winning ('No' $\rightarrow$ 'Yes', etc.) that we assume for any dichotomous rule based on such voting profiles:

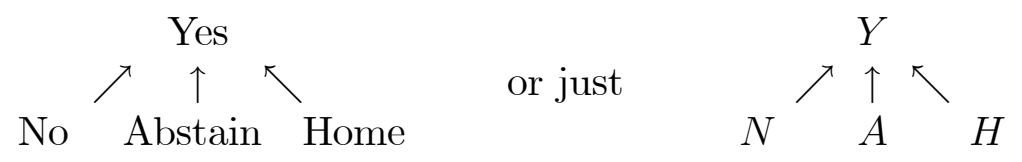

Most real world voting rules satisfy further monotonicities (see the next section). Nevertheless we include only these basic ones in the basic definition of voting rule in order to have a sufficiently general notion.

Now let us consider the extension of the other two properties satisfied by binary rules for a set $\mathcal{W} \subseteq 4^{\mathcal{N}}$. Full support of a proposal should imply its acceptance, thus we impose: 
Full-support: A set $\mathcal{W} \subseteq 4^{\mathcal{N}}$ is said to satisfy the full-support condition if a unanimous 'yes' leads to the acceptance of the proposal: If $S^{Y}=\mathcal{N}$, then $S \in \mathcal{W}$.

As to the extension of 'null-support' condition the situation is more delicate. An obvious extension is this:

Null-support: A set $\mathcal{W} \subseteq 4^{\mathcal{N}}$ is said to satisfy the null-support condition if the proposal is rejected in case of null support: If $S^{Y}=\varnothing$, then $S \notin \mathcal{W}$.

Nevertheless, if we want to avoid clashes with some configurations of monotonicities that are to be found in the specification of some real world voting rules, then this condition is too strong. For instance a participation quorum requirement considers as equivalent the options of voting 'yes', 'no' and abstaining. In the corresponding rule a configuration where $S^{Y}=\varnothing$ (but $S^{A} \neq \varnothing$ ) could be winning. Thus we weaken the 'null-support' condition in order to avoid ruling out such rules ${ }^{4}$. To that end we need a previous notion. Let $X, Z$ be two options, i.e. $X, Z \in\{Y, A, H, N\}$, and $\mathcal{W} \subseteq 4^{\mathcal{N}}$. We say that $X$ and $Z$ are 'equivalent in $\mathcal{W}$ ' and write $X \equiv_{\mathcal{W}} Z$, if for all $S \in 4^{\mathcal{N}}$

$$
S \in \mathcal{W} \Rightarrow T \in \mathcal{W} \text {, for all } T \in 4^{\mathcal{N}} \text { s.t. }
$$

$S^{X} \cup S^{Z}=T^{X} \cup T^{Z}$, and $S^{V}=T^{V}$ for all action $V \in\{Y, A, H, N\} \backslash\{Z, X\}$.

Now we can formulate a weak version of 'null-support' for this type of rule:

Weak null-support: A set $\mathcal{W} \subseteq 4^{\mathcal{N}}$ is said to satisfy the weak null-support condition if for all $S \in \mathcal{W}$, either $S^{Y} \neq \varnothing$ or there exists $X \in\{A, H, N\}$ s.t. $X \equiv_{\mathcal{W}} Y$ and $S^{X} \neq \varnothing$.

This condition is obviously weaker than 'null-support' and equivalent when no action is equivalent to voting 'yes'. Adding this condition and that of 'full-support' to the above monotonicities we define what in the sequel we refer to as a 'quaternary' dichotomous rule.

Definition 2 An n-voter 'quaternary dichotomous voting rule' ( $Q V R$ ) is a set $\mathcal{W}$ of 4-partitions of $\mathcal{N}$ that satisfies full-support, weak null-support, $N Y$-monotonicity, $A Y$ monotonicity and HY-monotonicity.

Remark: Given the monotonicities assumed, the 'full-support' condition can be replaced by this:

Nonemptiness: $\mathcal{W} \neq \varnothing$.

\footnotetext{
${ }^{4}$ In real world rules a participation quorum is usually associated with other requirements that break the equivalence between the 'yes' option and the others.
} 
The following preorders (i.e. binary reflexive and transitive relations) on the set of vote configurations can be naturally associated with each of the three basic monotonicities assumed:

$$
\begin{aligned}
& S \preceq_{N Y} T \Leftrightarrow\left(S^{Y} \subseteq T^{Y}, S^{A}=T^{A} \text { and } S^{H}=T^{H}\right), \\
& S \preceq_{A Y} T \Leftrightarrow\left(S^{Y} \subseteq T^{Y}, S^{N}=T^{N} \text { and } S^{H}=T^{H}\right), \\
& S \preceq_{H Y} T \Leftrightarrow\left(S^{Y} \subseteq T^{Y}, S^{A}=T^{A} \text { and } S^{N}=T^{N}\right),
\end{aligned}
$$

By means of these relations, by just replacing ' $X Y$ ' by the desired monotonicity (i.e. $N Y, A Y$ or $H Y$ ), the three monotonicities assumed can be expressed in the form: A rule $\mathcal{W}$ verifies $X Y$-monotonicity if

$$
\left(S \in \mathcal{W} \text { and } S \preceq_{X Y} T\right) \Rightarrow T \in \mathcal{W},
$$

entirely analogous to the monotonicity condition for binary rules: preorder ' $\preceq_{X Y}$ ' merely replaces ' $S^{Y} \subseteq T^{Y}$ '.

As has been done for each of these monotonicities separately, when all three of them are assumed it is possible to formulate all their implications by means of a single preorder $\preceq_{Q V R}$, given by the transitive closure of the union of the preorders associated with each of the three monotonicities, as stated explicitly by the following:

Definition 3 Given two vote configurations, $S$ and $T, S \preceq_{Q V R} T$ if and only if there exists a finite sequence of vote configurations $S_{1}, S_{2}, \ldots, S_{k}$ such that $S_{1}=S, S_{k}=T$ and for all $j=1,2, . ., k-1: S_{j} \preceq_{X Y} S_{j+1}$, where ' $\preceq_{X Y}$ ' is any of the relations defined by (1), (2) or (3).

Now it is possible to express the three monotonicities by a single implication which yields the following alternative definition of QVR:

Definition 4 An n-voter quaternary voting rule is a nonempty set $\mathcal{W}$ of 4-partitions of $\mathcal{N}$ that satisfies weak null-support and such that

$$
\left(S \in \mathcal{W} \text { and } S \preceq_{Q V R} T\right) \Rightarrow T \in \mathcal{W},
$$

where $\preceq_{Q V R}$ is the relation given by Definition 3.

Preorder $\preceq_{Q V R}$ can also be formulated exclusively in terms of configurations $S$ and $T$ as the following proposition shows:

Proposition 5 For any two vote configurations $S$ and $T$ :

$$
S \preceq_{Q V R} T \Leftrightarrow\left\{\begin{array}{l}
S^{N} \supseteq T^{N} \\
S^{H} \supseteq T^{H} \\
S^{A} \supseteq T^{A} .
\end{array}\right.
$$


Proof: $(\Rightarrow)$ Assume $S_{1}, S_{2}, \ldots, S_{k}$ are such that $S_{1}=S, S_{k}=T$ and for all $j=$ $1,2, . ., k-1: S_{j} \preceq_{X Y} S_{j+1}$. Then, from (1), (2) and (3), it can thus immediately be concluded that for any $j=1,2, . ., k-1$, we have: $S_{j}^{N} \supseteq S_{j+1}^{N}, S_{j}^{H} \supseteq S_{j+1}^{H}$ and $S_{j}^{A} \supseteq S_{j+1}^{A}$. Thus the three inclusions hold for $S$ and $T$.

$(\Leftarrow)$ Now, reciprocally, assume that the three inclusions hold for $S$ and $T$. Then let $S_{1}, S_{2}, S_{3}$ and $S_{4}$ be the following configurations: $S_{1}=S ; S_{2}$ such that

$$
S_{2}^{Y}=S_{1}^{Y} \cup\left(S_{1}^{A} \backslash T^{A}\right), S_{2}^{H}=S^{H}, S_{2}^{A}=T^{A}, S_{2}^{N}=S^{N}
$$

$S_{3}$ such that

$$
S_{3}^{Y}=S_{2}^{Y} \cup\left(S^{H} \backslash T^{H}\right), S_{3}^{H}=T^{H}, S_{3}^{A}=T^{A}, S_{3}^{N}=S^{N}
$$

and $S_{4}=T$. Then we have

$$
S=S_{1} \preceq_{A Y} S_{2} \preceq_{H Y} S_{3} \preceq_{N Y} S_{4}=T
$$

The latter because

$$
S_{4}^{Y}=S_{3}^{Y} \cup\left(S^{N} \backslash T^{N}\right), S_{3}^{H}=T^{H}, S_{3}^{A}=T^{A}, S_{3}^{N}=T^{N},
$$

that is, $S_{3} \preceq_{N Y} S_{4}=T$. Therefore $S \preceq_{Q V R} T$.

The preorder $\preceq_{Q V R}$ allows us to formulate the notion of 'minimal' vote configuration (relative to the monotonicities summarized by $\preceq_{Q V R}$ ), i.e. those winning configurations whose winning character cannot be inferred from that of other configurations and the three monotonicity conditions.

Definition 6 A vote configuration $S \in 4^{\mathcal{N}}$ is 'minimal winning' in rule $\mathcal{W}$ w.r.t. $\preceq_{Q V R}$ if $S \in \mathcal{W}$ and there is no other winning configuration $T$ such that $T \prec_{Q V R} S$ (i.e. such that $T \preceq_{Q V R} S$ but $S \varliminf_{Q V R} T$ ); and $S$ is 'maximal losing' w.r.t. $\preceq_{Q V R}$ if $S \notin \mathcal{W}$ and there is no other losing configuration $T$ such that $S \prec_{Q V R} T$.

A rule is anonymous if a vote configuration is winning or not dependent solely on the number of voters of each type.

Definition 7 A quaternary dichotomous voting rule is 'anonymous' if for all $S \in \mathcal{W}$ and all $T$ such that $t^{Y}=s^{Y}, t^{N}=s^{N}, t^{A}=s^{A}$ and $t^{H}=s^{H}$, we have $T \in \mathcal{W}$.

When the rule is anonymous the inclusions (5) that define the binary relation $\preceq_{Q V R}$ can be replaced by a set of inequalities involving the cardinalities of the sets of different types of vote. It is enough to replace each set by its cardinality (e.g. $S^{Y}$ by $s^{Y}$, etc.), and replacing ' $\subseteq$ ' by ' $\leq$ '. Thus, we have the following self-contained definition: 
Definition 8 An anonymous quaternary voting rule is a nonempty set $\mathcal{W}$ of 4 -partitions of $\mathcal{N}$ that satisfies weak null-support and such that

$$
\left(S \in \mathcal{W} \text { and } S \preceq_{A n Q V R} T\right) \Rightarrow T \in \mathcal{W}
$$

where $\preceq_{A n Q V R}$ is the relation given by

$$
S \preceq_{A n Q V R} T \Leftrightarrow\left\{\begin{array}{l}
s^{N} \geq t^{N} \\
s^{H} \geq t^{H} \\
s^{A} \geq t^{A} .
\end{array}\right.
$$

Finally, the notion of 'proper' rule remains to be extended to this wider class of rules, but we postpone this to the next section.

\section{The lattice of classes of monotonic QVRs}

All real world dichotomous voting rules based on the four options satisfy the three monotonicities considered so far, and consequently fit into the above general definition. Nevertheless, actual dichotomous voting rules often satisfy further monotonicities.

Definition 9 Given any two options $X, Z \in\{Y, A, H, N\}$, a $Q V R \mathcal{W}$ is $X Z$-monotonic if

$$
S \in \mathcal{W} \Rightarrow T \in \mathcal{W} \text { for all } T \text { s.t. } S \preceq_{X Z} T
$$

where

$$
S \preceq_{X Z} T \Leftrightarrow\left(S^{Z} \subseteq T^{Z}, \text { and } S^{V}=T^{V} \text { for } V \in\{Y, A, H, N\} \backslash\{Z, X\}\right) \text {. }
$$

By assuming different combinations of monotonicities (in addition to the three basic ones), a complete lattice of subclasses of QVRs related by inclusion arises. In the next section we constrain our attention to a sublattice of this excesively wide lattice by considering only some 'reasonable' combinations of monotonicities. Nevertheless it is convenient first to establish a few basic facts about the general lattice that will be useful later.

In what follows we refer to subclasses of $n$-voter QVRs (denoted by $C, C_{1}, C_{2}$ etc.) monotonic in the following sense:

Definition 10 A class of 'monotonic' $Q V R$ s is a class of QVRs that contains all QVRs that satisfy a specific set of $X Z$-monotonicities that includes the three basic ones.

Note that as a class $C$ of monotonic QVRs is characterized by a set of monotonicities, all the monotonicities within the class can be summarized in the form:

$$
\left(S \in \mathcal{W} \text { and } S \preceq_{C} T\right) \Rightarrow T \in \mathcal{W}
$$


where $\preceq_{C}$ is the preorder determined by the monotonicities that characterize $C$, as has been done for the whole class of QVRs. As in the case of $\preceq_{Q V R}$, it is given by the transitive closure of the union of the preorders associated with each of those monotonicities. In the next section some monotonic classes and their associated preorders are explicitly specified, but here we provide a list of all possible 'constellations' of monotonicities. In order to simplify the list, we use the following notation $\{V, X, Z\}=\{A, H, N\}$, and ' $X \rightarrow Z$ ' means that $X Z$-monotonicity holds, while ' $X \equiv Z$ ' means that these options are interchangeable as both $X \rightarrow Z$ and $Z \rightarrow X$ hold. In the diagrams we omit those monotonicities which are implied by those explicit: for instance, in $V \equiv X \rightarrow Z \rightarrow Y$ the arrow $X \rightarrow Y$ (and another 2) is omitted as implied by $X \rightarrow Z$ and $Z \rightarrow Y$. In this way, for instance, the pattern of monotonicities $V \equiv X \rightarrow Z \rightarrow Y$ represents three possible variations depending on whether $Z=A$ or $Z=H$ or $Z=N$. These are the possible configurations of monotonicities specifying a monotonic class:

-With (essentially) only one option there is a unique possibility:

$$
Y \equiv A \equiv H \equiv N
$$

-With up to two (essentially) different options there are three possible configurations:

$\begin{array}{ccc}Y & Y \equiv Z & Y \equiv Z \equiv X \\ \uparrow & \uparrow & \uparrow \\ A \equiv H \equiv N & X \equiv V & V\end{array}$

-With up to three (essentially) different options there are five possibilities:

\begin{tabular}{|c|c|c|c|c|}
\hline$Y$ & $Y$ & $Y \equiv Z$ & & \\
\hline$\uparrow$ & $\uparrow$ & $\uparrow$ & $Y$ & $Y \equiv Z$ \\
\hline$Z$ & $Z \equiv X$ & $X$ & & $\nearrow \nwarrow$ \\
\hline$\uparrow$ & $\uparrow$ & $\uparrow$ & $Z \equiv X$ & $X$ \\
\hline$\equiv V$ & $V$ & V & & \\
\hline
\end{tabular}

-With up to four different options there are five possibilities:
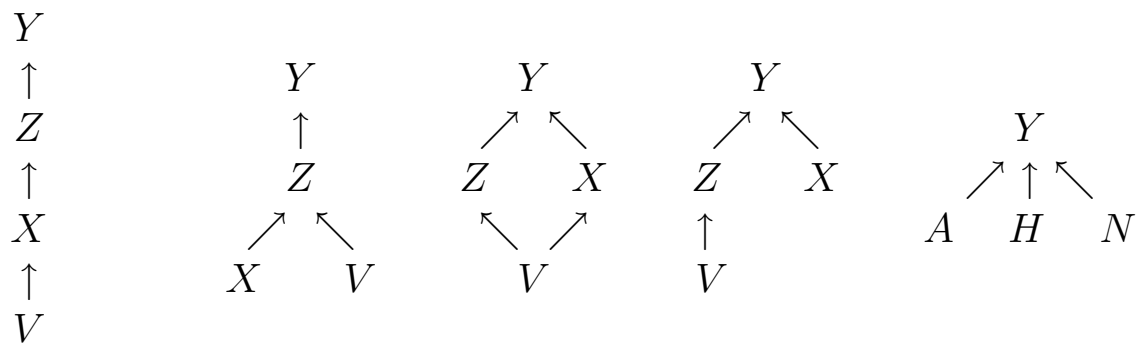

Observe that each monotonic class $C$ is specified by a combination of monotonicities that fits one of the above diagrams, which can be seen as a preorder $\leq_{C}$ over the set of options $\{Y, A, H, N\}$ (that determines a preorder $\preceq_{C}$ over vote configurations). In some cases the preorder over the set of options is linear (i.e. it is complete and transitive). 
This is the case for all monotonicity configurations with only one or two options, but also for the first three of those with 3 options and the first one with 4 options. In what follows we refer to such monotonic classes of QVRs as the 'linear classes' in reference to this linear preorder, and we refer as 'linear rules' to those that belong to any of these classes. Then we have the following definitions and facts.

Proposition 11 Let $C_{1}$ and $C_{2}$ be two classes of monotonic QVRs, then

$$
\left(C_{1} \subseteq C_{2}\right) \Leftrightarrow\left(\leq_{C_{1}} \supseteq \leq_{C_{2}}\right) \Leftrightarrow\left(\preceq_{C_{1}} \supseteq \preceq_{C_{2}}\right) .
$$

Proof. $C_{1} \subseteq C_{2}$ means that any monotonicity in $C_{2}$ holds also in $C_{1}$ (i.e., $\leq_{C_{2}} \subseteq$ $\leq_{C_{1}}$ ), and more monotonicities in $C_{1}$ means a richer preorder relationship, thus the conclusion follows straightforwardly.

It is important to remark that the notions of minimal winning and maximal losing configuration are relative to the preorder over vote configurations that summarizes the monotonicities that characterize the monotonic class within which we are working. That is, if $\preceq_{C}$ is the preorder associated with class $C$, we denote

$$
\begin{aligned}
& \operatorname{Minw}_{C}(\mathcal{W}):=\left\{S \in \mathcal{W}:\left(T \prec_{C} S \Rightarrow T \notin \mathcal{W}\right)\right\} . \\
& \operatorname{Maxl}_{C}(\mathcal{W}):=\left\{S \notin \mathcal{W}:\left(S \prec_{C} T \Rightarrow T \in \mathcal{W}\right)\right\} .
\end{aligned}
$$

Then we have the following relation.

Proposition 12 Given two classes of monotonic QVRs, $C_{1}$ and $C_{2}$, if $\mathcal{W} \in C_{1} \subseteq C_{2}$, then $\operatorname{Minw}_{C_{1}}(\mathcal{W}) \subseteq \operatorname{Minw}_{C_{2}}(\mathcal{W})$ and $\operatorname{Maxl}_{C_{1}}(\mathcal{W}) \subseteq \operatorname{Maxl}_{C_{2}}(\mathcal{W})$.

Proof. Assume $\mathcal{W} \in C_{1} \subseteq C_{2}$. Let $S \in \operatorname{Minw}_{C_{1}}(\mathcal{W})$, if $T \prec_{C_{2}} S$, then, in view of Proposition $11, T \prec_{C_{1}} S$, and consequently $T \notin \mathcal{W}$. Therefore $S \in \operatorname{Minw}_{C_{2}}(\mathcal{W})$. The other inclusion is proved in the same way.

Definition 13 Given two classes of monotonic $Q V R s, C_{1}$ and $C_{2}$, we call their 'meet', and denote by $C_{1} \wedge C_{2}$, the class of monotonic QVRs that satisfy all the monotonicities in $C_{1}$ and all the monotonicities in $C_{2}$, and call their 'join' and denote by $C_{1} \vee C_{2}$ the class of monotonic QVRs that satisfy all the monotonicities that hold both in $C_{1}$ and in $C_{2}$.

In fact ' $\wedge$ ' (' $\vee$ ') gives the greatest (least) lower (upper) bound of any two classes in the complete lattice of all monotonic classes partially ordered by inclusion. The minimal element in the lattice is the class where all monotonicities hold, which contains only the degenerated rule where all vote configurations are winning (first in the list of classes given above). Note that this rule satisfies 'full-support' and 'weak null-support'. The maximal class is that of all QVRs (last in the list given above). Meet and join are related with union and intersection by the following proposition, whose simple proof is omitted. 
Proposition 14 Let $C_{1}$ and $C_{2}$ be two monotonic classes of $Q V R s$, then: (i) $C_{1} \wedge C_{2}=$ $C_{1} \cap C_{2}$ and $\preceq_{C_{1} \wedge C_{2}}$ is the transitive closure of $\preceq_{C_{1}} \cup \preceq_{C_{2}}$; and (ii) $C_{1} \vee C_{2} \supseteq C_{1} \cup C_{2}$ and $\preceq_{C_{1} \vee C_{2}}=\preceq_{C_{1}} \cap \preceq_{C_{2}}$.

The union and the intersection are means of defining new rules from preexisting ones, and we are interested in how properties and these operations interact. Let us examine first the basic conditions and then the monotonicities.

Proposition 15 Given $\mathcal{W}_{1}, \mathcal{W}_{2} \subseteq 4^{\mathcal{N}}$, then:

(i) If both $\mathcal{W}_{2}$ and $\mathcal{W}_{2}$ satisfy 'full-support', then $\mathcal{W}_{1} \cup \mathcal{W}_{2}$ and $\mathcal{W}_{1} \cap \mathcal{W}_{2}$ also satisfy it. Moreover, even if only one of them satisfies 'full-support' then $\mathcal{W}_{1} \cup \mathcal{W}_{2}$ also satisfies it.

(ii) If both $\mathcal{W}_{1}$ and $\mathcal{W}_{2}$ satisfy 'null-support', then $\mathcal{W}_{1} \cup \mathcal{W}_{2}$ and $\mathcal{W}_{1} \cap \mathcal{W}_{2}$ also satisfy it. Moreover, even if only one of them satisfies 'null-support' then $\mathcal{W}_{1} \cap \mathcal{W}_{2}$ also satisfies it.

(iii) If both satisfy 'weak null-support' $\mathcal{W}_{1} \cup \mathcal{W}_{2}$ also satisfies it, but $\mathcal{W}_{1} \cap \mathcal{W}_{2}$ may fail to satisfy it.

Proof. Parts (i) and (ii) are straightforward.

(iii) Assume that $\mathcal{W}_{1}$ and $\mathcal{W}_{2}$ satisfy 'weak null-support'. If $S \in \mathcal{W}_{1} \cup \mathcal{W}_{2}$, then either $S \in \mathcal{W}_{1}$ or $S \in \mathcal{W}_{2}$. As both rules satisfy 'weak null-support', we have that either

$$
(S \neq \varnothing) \text { or }\left(\exists X \equiv_{\mathcal{W}_{1}} Y \text { s.t. } S^{X} \neq \varnothing\right)
$$

or

$$
(S \neq \varnothing) \text { or }\left(\exists Z \equiv_{\mathcal{W}_{2}} Y \text { s.t. } S^{Z} \neq \varnothing\right) .
$$

Therefore, either $S \neq \varnothing$ or

$$
\left(\exists X \equiv \mathcal{W}_{1} Y \text { s.t. } S^{X} \neq \varnothing\right) \text { or }\left(\exists Z \equiv \mathcal{W}_{2} Y \text { s.t. } S^{Z} \neq \varnothing\right) .
$$

In the latter case assume, for instance, that $\exists X \equiv_{\mathcal{W}_{1}} Y$ s.t. $S^{X} \neq \varnothing$, then $X \equiv_{\mathcal{W}_{1} \cup \mathcal{W}_{2}} Y$ and $S^{X} \neq \varnothing$, so that $\mathcal{W}_{1} \cup \mathcal{W}_{2}$ satisfies 'weak null-support'.

The following counterexample shows that $\mathcal{W}_{1} \cap \mathcal{W}_{2}$ may fail to satisfy 'weak nullsupport'. Let $\mathcal{W}_{1}$ and $\mathcal{W}_{2}$ be the 30 -voters rules specified by

$$
\mathcal{W}_{1}=\left\{S \in 4^{\mathcal{N}}:\left(s^{Y}+s^{A}+0.5 s^{H}>s^{N}\right) \&\left(s^{Y}+s^{A}>9\right)\right\}
$$

and

$$
\mathcal{W}_{2}=\left\{S \in 4^{\mathcal{N}}:\left(s^{Y}+s^{H}+0.5 s^{A}>s^{N}\right) \&\left(s^{Y}+s^{H}>9\right)\right\} .
$$

Both rules satisfy 'weak null-support', in $\mathcal{W}_{1}$ we have $A \equiv_{\mathcal{W}_{1}} Y$, and in $\mathcal{W}_{2}$ it holds that $H \equiv \mathcal{W}_{2} Y$, while in $\mathcal{W}_{1} \cap \mathcal{W}_{2}$ we have $\nexists Z \equiv_{\mathcal{W}_{1} \cap \mathcal{W}_{2}} Y$. Then for any configuration $S=\left(S^{Y}, S^{A}, S^{H}, S^{N}\right)$ s.t. $s^{Y}=0$, and $s^{A}=s^{H}=s^{N}=10$, we have

$$
S \in \mathcal{W}_{1} \cap \mathcal{W}_{2}
$$

$S^{Y}=\varnothing$, and $\nexists Z \equiv_{\mathcal{W}_{1} \cap \mathcal{W}_{2}} Y$. Thus, $\mathcal{W}_{1} \cap \mathcal{W}_{2}$ does not satisfy 'weak null-support'.

As to the monotonicities we have: 
Proposition 16 Let $C_{1}$ and $C_{2}$ be two classes of $Q V R$ s specified by sets of monotonicities with associated preorders $\preceq_{C_{1}}$ and $\preceq_{C_{2}}$. If $\mathcal{W}_{1} \in C_{1}$, and $\mathcal{W}_{2} \in C_{2}$, then $\mathcal{W}_{1} \cup \mathcal{W}_{2} \in C_{1} \wedge C_{2}$, and, if $\mathcal{W}_{1} \cap \mathcal{W}_{2}$ satisfies 'weak null-support', $\mathcal{W}_{1} \cap \mathcal{W}_{2} \in C_{1} \vee C_{2}$.

Proof. Just observe that $\mathcal{W}_{1} \cap \mathcal{W}_{2}$ satisfies those monotonicities that are satisfied by both $\mathcal{W}_{1}$ and $\mathcal{W}_{2}$, while $\mathcal{W}_{1} \cup \mathcal{W}_{2}$ satisfies all those satisfied by $\mathcal{W}_{1}$ and all those satisfied by $\mathcal{W}_{2}$. Then by Prop. 14 the conclusion follows, but note that in the light of Prop. 15 'weak null support' is not guaranteed for $\mathcal{W}_{1} \cap \mathcal{W}_{2}$, hence the if clause in the statement about $\mathcal{W}_{1} \cap \mathcal{W}_{2}$.

In section 5 we address the representability of QVRs by means of weighted rules. The following result will be useful there:

Proposition 17 Let $C_{1}$ and $C_{2}$ be two monotonic classes of $Q V R$ s and $C=C_{1} \vee C_{2}$, then for all $\mathcal{W} \in C$ there exist $\mathcal{W}_{1} \in C_{1}$ and $\mathcal{W}_{2} \in C_{2}$ such that $\mathcal{W}=\mathcal{W}_{1} \cap \mathcal{W}_{2}$.

Proof. Let $\preceq_{C}$ denote the preorder associated with class $C$ and $\mathcal{W} \in C$, so that we have:

$$
\mathcal{W}=\left\{T \in 4^{\mathcal{N}}: S \preceq_{C} T \text { for some } S \in \operatorname{Minw}_{C}(\mathcal{W})\right\} .
$$

Let $\preceq_{C_{1}}$ and $\preceq_{C_{2}}$ denote the preorders associated with $C_{1}$ and $C_{2}$. Let $\mathcal{W}_{1}$ and $\mathcal{W}_{2}$ be the rules defined by:

$$
\mathcal{W}_{1}=\left\{T \in 4^{\mathcal{N}}: S \preceq C_{1} T \text { for some } S \in \operatorname{Minw}_{C}(\mathcal{W})\right\}
$$

and

$$
\mathcal{W}_{2}=\left\{T \in 4^{\mathcal{N}}: S \preceq C_{2} T \text { for some } S \in \operatorname{Minw} w_{C}(\mathcal{W})\right\} .
$$

Then, as can easily be checked, $\mathcal{W}_{1}$ and $\mathcal{W}_{2}$ satisfy 'full-support' and 'weak nullsupport'. Thus, obviously, $\mathcal{W}_{1} \in C_{1}, \mathcal{W}_{2} \in C_{2}$, and we have

$$
\begin{aligned}
\mathcal{W}_{1} \cap \mathcal{W}_{2} & =\left\{T \in 4^{\mathcal{N}}: S \preceq_{C_{1}} T, S \preceq_{C_{2}} T \text { for some } S \in \operatorname{Minw}_{C}(\mathcal{W})\right\} \\
& =\left\{T \in 4^{\mathcal{N}}: S \preceq^{*} T \text { for some } S \in \operatorname{Minw}_{C}(\mathcal{W})\right\}
\end{aligned}
$$

where $\preceq^{*}=\preceq_{C_{1}} \cap \preceq_{C_{2}}$, and as $C=C_{1} \vee C_{2}$, by Prop. 14 we have, $\preceq_{C_{1}} \cap \preceq_{C_{2}}=\preceq_{C}$. Then we conclude

$$
\mathcal{W}_{1} \cap \mathcal{W}_{2}=\left\{T \in 4^{\mathcal{N}}: S \preceq_{C} T \text { and } S \in \operatorname{Minw_{C}}(\mathcal{W})\right\}=\mathcal{W}
$$

We now extend the notion of 'proper' rule to the class of QVRs. The point of this condition, satisfied by all binary rules by means of which issues of substance are decided upon, is to prevent two disjoint groups of voters with opposed preferences from both being winning when supporting opposite proposals. The difficulty of extending this condition to QVRs is that for real world rules that can be expressed as QVRs it is often the case that two disjoint groups of voters can win a vote if a sufficient number of 
voters abstain and/or do not turn out. For instance, if only more 'yes' than 'no' voters is required in addition to a certain quorum (i.e. a certain maximal number of stayingat-home voters) to pass a decision, this may happen. But no real problem arises, as it is the result of admitting abstention and staying at home as legitimate options for possibly indifferent voters. The problem arises if this may also happen in cases where all voters have strict preferences either for approval or rejection of a proposal. This motivates the following

Definition 18 A set of voters $R \subseteq \mathcal{N}$ is 'strong winning' for a $Q V R \mathcal{W} \subseteq 4^{\mathcal{N}}$ if for all $S \in 4^{\mathcal{N}}$ such that $S^{Y}=R$ we have $S \in \mathcal{W}$.

Definition 19 Let $\mathcal{W} \subseteq 4^{\mathcal{N}}$ be a $Q V R$, the 'core binary rule' associated with $\mathcal{W}$ is the binary rule (which we denote by $\mathcal{V}_{\mathcal{W}}^{*}$ )

$$
\mathcal{V}_{\mathcal{W}}^{*}:=\left\{T \in 2^{\mathcal{N}}: T^{Y} \text { is strong winning in } \mathcal{W}\right\}
$$

That this definition is sound is proved by the following

Proposition 20 For any non degenerated $Q V R, \mathcal{W}$, its associated core binary rule $\mathcal{V}_{\mathcal{W}}^{*}$ is actually a binary voting rule.

Proof. Let $\mathcal{W}$ be a non degenerated QVR. Full-support of $\mathcal{W}$ ensures that $(\mathcal{N}, \varnothing) \in$ $\mathcal{V}_{\mathcal{W}}^{*}$. Now assume that $(\varnothing, \mathcal{N}) \in \mathcal{V}_{\mathcal{W}}^{*}$. In this case, from the three basic monotonicities, it is straightforward to check that for all $S \in 4^{\mathcal{N}}, S \in \mathcal{W}$. In other words, $\mathcal{W}$ is the degenerated rule. Thus, $\mathcal{V}_{\mathcal{V}}^{*}$ satisfies full support and null support. Finally, if $T, Q \in 2^{\mathcal{N}}$, with $T \in \mathcal{V}_{\mathcal{W}}^{*}$ and $T^{Y} \subseteq Q^{Y}$, then the basic monotonicities of $\mathcal{W}$ imply that $Q \in \mathcal{V}_{\mathcal{W}}^{*}$. Thus $\mathcal{V}_{\mathcal{W}}^{*}$ is a binary voting rule.

Alternatively, the notion of the associated core rule can also be formulated as a QVR:

Definition 21 The 'core $Q V R$ ' associated with a $Q V R \mathcal{W}$ is the $Q V R$

$$
\mathcal{W}^{*}:=\left\{S \in 4^{\mathcal{N}}: S^{Y} \text { is strong winning in } \mathcal{W}\right\} .
$$

Observe that in this formulation $\mathcal{W}^{*} \subseteq \mathcal{W}$. In fact, the core rule $\mathcal{W}^{*}$ is the maximal QVR in the class

$$
\begin{gathered}
Y \\
\uparrow \\
A \equiv H \equiv N
\end{gathered}
$$

contained in $\mathcal{W}$, which motivates the term 'core' rule. Now we can formulate a sensible notion of properness for QVRs consistent with the usual notion for binary rules.

Definition 22 A quaternary voting rule $\mathcal{W}$ is 'proper' if the associated binary core rule is proper.

The following straightforward fact is interesting when defining rules by intersection of others.

Proposition 23 The intersection of two QVRs is proper if at least one of them is proper. 


\section{Some examples and classes of QVRs rules}

The preceding section adopted a very general point of view for a general study the lattice of classes of monotonic QVRs. Now, in order to form a picture of the main subclasses of such rules, we consider some reasonable combinations of monotonicities. In this way we obtain a sublattice of the general lattice.

Solely on the basis of the three monotonicities assumed, the options of staying at home, voting 'no' and abstaining cannot be compared in terms of being more or less preferable for the acceptance or rejection of the proposal. In most actual rules abstention is at least as good as voting 'no' for the acceptance of the proposal. It is then reasonable to consider rules that verify $N A$-monotonicity (for a precise definition just replace ' $X Z{ }^{-}$' by ' $N A$-' in Def. 9). If we add this condition to the three basic ones, we specify a subclass of ' $N A$-monotonic' rules, whose associated diagram and preorder are

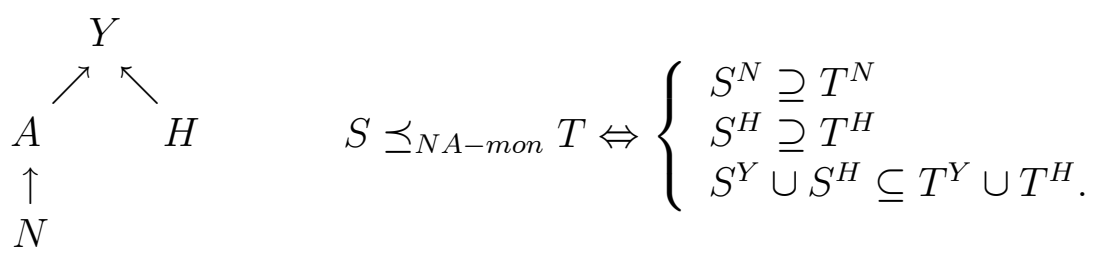

As a particular case one may have $N A$-equivalence. In some rules (for instance, the majority of present voters with a participation quorum) the option of staying at home and the 'no'-option cannot be compared in terms of being more or less preferable for the acceptance or rejection of the proposal. In the rules where these options can be compared, it is reasonable to assume that staying at home is at least as favorable to a final 'yes' as voting 'no', that is, $N H$-monotonicity. Strict $H N$-monotonicity is thus ruled out, $H N$-monotonicity is admitted only together with $N H$-monotonicity, which results in $\mathrm{NH}$-equivalence. We also rule out from the sublattice any rule that would display $Y X$-monotonicity $X \in\{A, H, N\}$. Indeed in practice the 'yes'-option is always strictly more favorable to the acceptance of the proposal than any other option.

Considering the different combinations of the above monotonicities and equivalences, we obtain the following nine classes of monotonic QVRs. For each class we give: (i) A diagram that represents the preorder $\leq_{C}$ over options that specifies its monotonicities; (ii) The preorder $\preceq_{C}$ over vote configurations associated with the monotonicities specific to the class (in all cases we express the relation $S \preceq_{C} T$ directly in terms of the involved configurations $S$ and $T$ and omit the proof, which in all cases is similar to that of Proposition 5, in this way a self-contained definition of each class of QVRs in similar terms to those of Def. 4 can be obtained merely by replacing $S \preceq_{Q V R} T$ by $S \preceq_{C} T$ ); and (iii) One or more examples (from the real world whenever we have found any) for which such a class is the smallest (in the lattice of monotonic classes) that contains it. 
(C1) NA-monotonic and $H A$-monotonic QVRs:

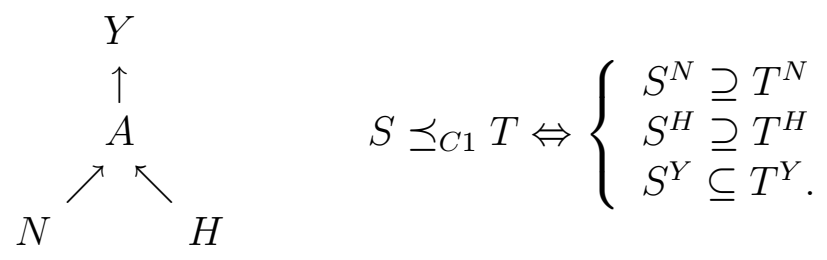

Example: A simple majority with a participation quorum

$$
\mathcal{W}=\left\{S \in 4^{\mathcal{N}}:\left(s^{Y}>s^{N}\right) \&\left(s^{Y}+s^{N}+s^{A}>\frac{n}{2}\right)\right\}
$$

is used in Parliaments such as those of Belgium and Italy.

(C2) NA-monotonic and $N H$-monotonic QVRs:

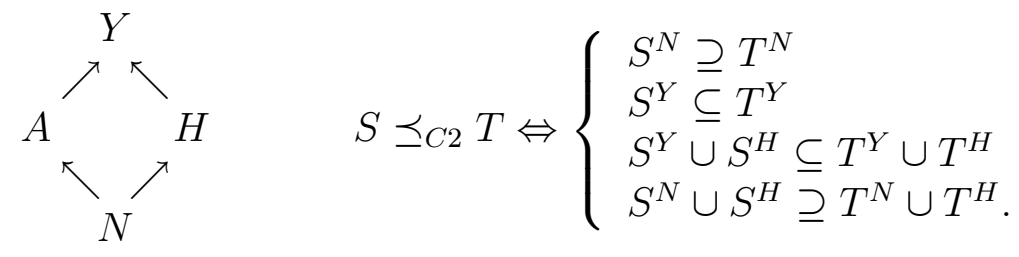

Example: $\mathcal{W}=\left\{S \in 4^{\mathcal{N}}:\left(s^{Y}>s^{N}+\frac{s^{H}}{2}\right) \&\left(s^{Y}>s^{N}+\frac{s^{A}}{2}\right)\right\}$.

(C3) NA-equivalent QVRs:

$$
\stackrel{\nearrow_{N}^{Y} \nwarrow H}{A \equiv} \quad S \preceq_{C 3} T \Leftrightarrow\left\{\begin{array}{l}
S^{H} \supseteq T^{H} \\
S^{Y} \cup S^{H} \subseteq T^{Y} \cup T^{H} .
\end{array}\right.
$$

Example: A majority of present voters with a participation quorum

$$
\mathcal{W}=\left\{S \in 4^{\mathcal{N}}:\left(s^{Y}>\frac{s^{Y}+s^{A}+s^{N}}{2}\right) \&\left(s^{H}<\frac{n}{2}\right)\right\}
$$

is used in the Spanish and German Parliaments.

(C4) NHA-monotonic QVRs: We denote by 'NHA-monotonic' those QVRs which are $\mathrm{NH}$-monotonic and $H A$-monotonic. 
Example: $\mathcal{W}=\left\{S \in 4^{\mathcal{N}}: s^{Y}>s^{N}+\frac{s^{H}}{2}+\frac{s^{A}}{4}\right\}$.

(C5) NAH-monotonic QVRs:

$$
\begin{gathered}
Y \\
\uparrow \\
H \\
\uparrow \\
A \\
\uparrow \\
N
\end{gathered}
$$

$$
S \preceq_{C 5} T \Leftrightarrow\left\{\begin{array}{l}
S^{Y} \subseteq T^{Y} \\
S^{N} \supseteq T^{N} \\
S^{Y} \cup S^{H} \subseteq T^{Y} \cup T^{H}
\end{array}\right.
$$

Example: $\mathcal{W}=\left\{S \in 4^{\mathcal{N}}: s^{Y}>s^{N}+\frac{s^{A}}{2}+\frac{s^{H}}{4}\right\}$.

(C6) $N A$-monotonic and $N H$-equivalent QVRs:

$$
\begin{gathered}
Y \\
\uparrow \\
A \\
\uparrow \\
H \stackrel{\uparrow}{\equiv} N
\end{gathered}
$$

Example: $\mathcal{W}=\left\{S \in 4^{\mathcal{N}}: s^{Y}>\frac{s^{Y}+s^{N}+s^{H}}{2}\right\}$.

(C7) $N A$-monotonic and $H A$-equivalent QVRs:

$$
\underset{\uparrow}{A \underset{N}{\risingdotseq} H} \quad S \preceq_{C 7} T \Leftrightarrow\left\{\begin{array}{l}
S^{N} \supseteq T^{N} \\
S^{Y} \subseteq T^{Y} .
\end{array}\right.
$$

Example: A simple majority (with no quorum):

$$
\mathcal{W}=\left\{S \in 4^{\mathcal{N}}: s^{Y}>s^{N}\right\}
$$

is used in the Swedish Parliament.

(C8) $N A$-equivalent and $A H$-monotonic QVRs:

$$
\begin{aligned}
& Y \\
& \stackrel{\uparrow}{H} \\
& \stackrel{\uparrow}{\equiv} \\
& A N
\end{aligned} \quad S \preceq_{C 8} T \Leftrightarrow\left\{\begin{array}{l}
S^{Y} \subseteq T^{Y} \\
S^{Y} \cup S^{H} \subseteq T^{Y} \cup T^{H} .
\end{array}\right.
$$

Examples: (a) A majority of members present

$$
\mathcal{W}=\left\{S \in 4^{\mathcal{N}}: s^{Y}>\frac{\left(s^{Y}+s^{N}+s^{A}\right)}{2}\right\}
$$


is used in the Finnish Parliament.

(b) A majority of those present with an 'approval' quorum

$$
\mathcal{W}=\left\{S \in 4^{\mathcal{N}}:\left(s^{Y}>\frac{s^{Y}+s^{N}+s^{A}}{2}\right) \&\left(s^{Y}>\frac{n}{4}\right)\right\}
$$

is used in the Greek Parliament.

(C9) NHA-equivalent QVRs (i.e. those which are $N H$-equivalent and $H A$-equivalent).

$$
\begin{gathered}
\uparrow \\
\uparrow \\
A
\end{gathered} \stackrel{H}{H} \quad S \preceq_{C 9} T \Leftrightarrow S^{Y} \subseteq T^{Y} .
$$

Example: An absolute 3/5-majority

$$
\mathcal{W}=\left\{S \in 4^{\mathcal{N}}: s^{Y} \geqslant \frac{3}{5} n\right\}
$$

is used in some Parliaments such as that of Estonia (in order to amend the Constitution) or that of Poland (to overrule the veto of the President).

Figure 1 represents the 9 subclasses of $N A$-monotonic quaternary dichotomous voting rules specified by the above combinations of monotonicity conditions considered. The upwards arrows represent the inclusion relation between different classes. In the light of Proposition 11, to prove a particular inclusion between two classes it suffices to check that the reciprocal inclusion holds for the given associated preorders, which is straightforward as the reader may see. These 9 classes along with the $N A$-monotonic class including them, related by inclusion, form a sublattice whose maximal element is the class of $N A$-monotonic rules and its minimal element is class $C 9$. The 'lack of symmetry' apparent in Figure 1 is due to the fact of having ruled out some monotonicities (for instance strict $\mathrm{HN}$-monotonicity as mentioned above) and consequently some monotonic classes as implausible. For the same reason, some pairs of classes have their intersection as their maximal lower bound in the sublattice is (e.g. classes $C 1$ and $C 2$ ) but others do not (e.g. classes $C 1$ and $C 3$ ).

The following comments are pertinent here:

\section{Remarks:}

(i) We provide real world examples of rules for which a given class is the smallest one containing it for only 5 classes in the sublattice $(C 1, C 3, C 7, C 8$, and $C 9)$, but the fact that two of these five cases $(C 1$ and $C 3)$ are not covered by the models in the literature shows that the extension in this paper is not a purely formal abstract exercise. Moreover, the picture provides a framework within which some of the extensions of binary rules to be found in the literature can be identified, as pointed out in subsequent remarks. Moreover, some classes for which no real world example is given prove important when we address the question of representability of QVRs by weighted voting rules. 


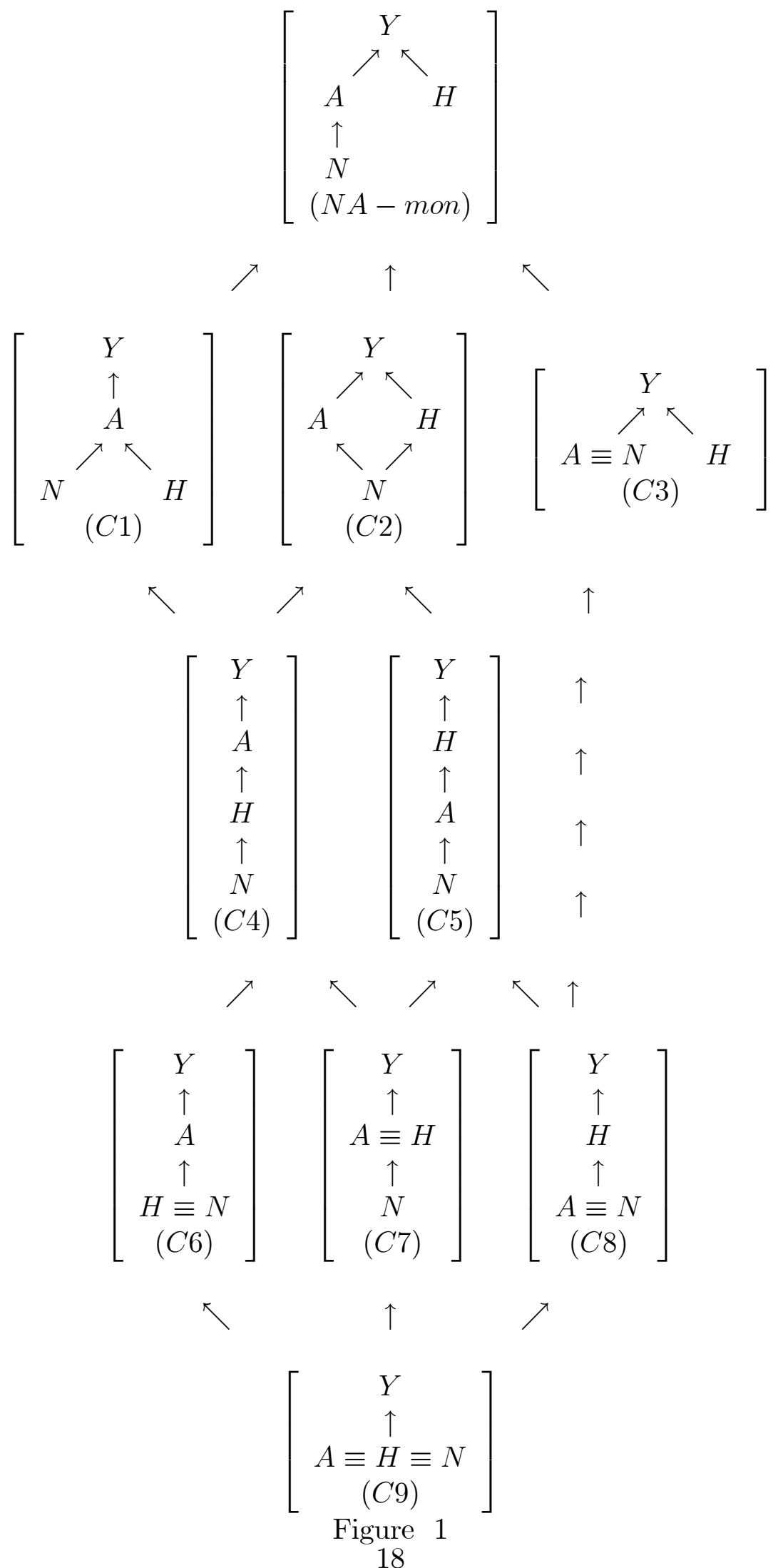


(ii) There is a case of a real world voting rule that does not belong to this sublattice which is worth commenting on. This is the voting rule that was used in the US Congress till $1890^{5}$, where abstaining could be more effective for rejection than voting 'no', a sort of simple majority with a 'votes-cast' quorum:

$$
\mathcal{W}=\left\{S \in 4^{\mathcal{N}}:\left(s^{Y}>s^{N}\right) \&\left(s^{Y}+s^{N}>\frac{n}{2}\right)\right\} .
$$

As it does not satisfy $N A$-monotonicity it is excluded from the sublattice considered here. This perhaps explains why the rule was abandoned and Speaker Thomas Reed replaced the 'votes-cast' quorum by a participation quorum. But observe that this rule belongs to the class

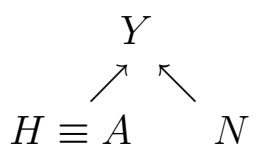

which satisfies the three basic monotonicities and therefore is a monotonic class of QVRs according to general Definition 2.

(iii) Similarly the rule that corresponds to a participation quorum requirement

$$
\mathcal{W}=\left\{S \in 4^{\mathcal{N}}: s^{Y}+s^{A}+s^{N}>\frac{n}{2}\right\}
$$

does not belong to any of the reasonable classes considered in the sublattice (although it also satisfies the three basic monotonicities of Definition 2). The monotonicity diagram and associated preorder are:

$$
Y \equiv \underset{\uparrow}{A} \equiv N \quad S \preceq_{\text {quorum }} T \Leftrightarrow T^{H} \subseteq S^{H} .
$$

Note that although such rules conflict with the 'null-support' condition (as this implies that some configuration where nobody votes 'yes' is winning) they satisfy 'weak nullsupport'.

(iv) In the 'minimal' class of the sublattice, $C 9$, where the number of monotonicities assumed is maximal in the sublattice, the binary relationship that summarizes them is maximal and the simplest: the inclusion between sets of 'yes'-voters. In fact, this class is isomorphic to that of classical binary voting rules. In other words, the general model of QVR (and this sublattice) includes the binary model as a particular case.

(v) The simplest as closest to the latter class are $C 6, C 7$ and $C 8$, where actually two options collapse by identification into one ( $H$ and $N$ in $C 6, H$ and $A$ in $C 7$, and $A$ and $N$ in $C 8$ ), thus leaving only three really different options. Felsenthal and Machover's (1997) 'ternary' voting rules correspond to classes $C 6$ and $C 7$ as they consider 'yes', 'no' and 'abstention' as separate options and the monotonicities that they assume are

\footnotetext{
${ }^{5}$ Source: Vermeule (2007).
} 
precisely the ones for these classes. Observe that the rules in class $C 3$ could also be called 'ternary' (as there are actually three options: $Y, N$ and $A \equiv H$ ) but they are not covered by the model considered by Felsenthal and Machover.

(vi) All the rules in this sublattice where monotonicities provide a linear order or linear preorder between the voters ${ }^{6}$ options (i.e. linear classes $C 4$ to $C 9$ ), can be seen as (4,2)-rules in the terms of Freixas and Zwicker (2003), or as ( $j, 2)$-rules with $j=4,3,2$, if we identify equivalent actions. Nevertheless the class of dichotomous rules that we consider is not included in Freixas and Zwicker's model of $(j, 2)$-voting rules because we do not require monotonicities to amount always to a linear preorder. Thus, $N A$-monotonic rules not contained in the 'smaller' classes $C 4$ to $C 9$, are not covered by their model. This includes many rules with quora, where voting 'no' or staying at home cannot really be compared, in the sense that in some configurations the 'no' option may be more favorable to the passage of the proposal, while in other configurations the opposite is true.

(vii) As with the general class of QVRs, for each of the monotonic classes considered the preorder associated with its subclass of anonymous rules can be specified by replacing each set by its cardinality (e.g. $S^{Y}$ by $s^{Y}$, etc.), ' $\subseteq$ ' by ' $\leq$ ' and ' $\cup$ ' by ' + ' in the different definitions. For instance, the binary relation associated with anonymous rules in the monotonic class $C 4$ is defined by

$$
S \preceq_{A n C 4} T \Leftrightarrow\left\{\begin{array}{l}
s^{Y} \leq t^{Y} \\
s^{N} \geq t^{N} \\
s^{N}+s^{H} \geq t^{N}+t^{H} .
\end{array}\right.
$$

Then, condition

$$
\left(S \in \mathcal{W} \text { and } S \preceq_{A n C 4} T\right) \Rightarrow T \in \mathcal{W},
$$

along with nonemptiness and 'weak null-support' specifies the anonymous subclass of $C 4$. All the the other classes can be similarly 'anonymized'.

\section{$5 \quad$ Weighted QVRs and dimension}

The simplest and best known binary dichotomous voting rules are weighted $q$-majority voting rules. In fact, most binary rules to be found in real-world collective decision bodies are either of this type or such that their sets of winning vote configurations are the intersections of the sets of winning configurations of two or more such rules. In this section, based on Freixas and Zwicker (2003), we extend the notion of weighted $q$-majority voting rule to the wider domain of quaternary voting rules and address the question of the representability of quaternary voting rules as (or by means of) such weighted quaternary $q$-majority voting rules.

A binary weighted majority rule is specified by a system of weights $\mathbf{w}=\left(w_{1}, . ., w_{n}\right)$, and a quota $Q>0$, so that the final result is 'yes' if the sum of the weights in favor of 
the proposal is larger than the quota. Denoting this rule by $\mathcal{B}(Q, \mathbf{w})$, we have:

$$
\mathcal{B}(Q, \mathbf{w})=\left\{S \in 2^{\mathcal{N}}: \sum_{i \in S^{Y}} w_{i}>Q\right\} .
$$

As is well known, not all binary voting rules can be represented in this way: some can only be represented by a double weighted majority, triple majority, etc. A $k$-multiple binary weighted majority rule is specified by $k$ systems of weights $\mathbf{w}_{r}=\left(w_{r 1}, . ., w_{r n}\right)$, where $w_{r i}$ represents the weight of voter $i$ in rule $r$, and $k$ quotas $Q_{r}(r=1,2, . ., k)$, each quota $Q_{r}$ corresponding to $\mathbf{w}_{r}$ system of weights. The final result is 'yes' if for all systems the sum of the weights in favor of the proposal is larger than the corresponding quota. The resulting rule is

$$
\bigcap_{r=1}^{r=k} \mathcal{B}\left(Q_{r}, \mathbf{w}_{r}\right)=\left\{S \in 2^{\mathcal{N}}: \sum_{i \in S^{Y}} w_{r i}>Q_{r}, \text { for all } r=1,2, . ., k\right\},
$$

Taylor and Zwicker $(1992,1993,1999)$ give necessary and sufficient conditions for a binary voting rule to be representable as a weighted majority rule, and introduce the notion of the dimension of a binary voting rule as the minimum number of weighted majority rules necessary to represent the voting rule (i.e. the minimal $k$ for which the rule can be expressed in the form (7). This notion is well defined as they prove that any binary voting rule can be represented in this way.

We now extend the notion of weighted majority rule to the class of quaternary voting rules dealt with in this paper. We use the following notation:

Definition 24 For any two vectors in $\mathbb{R}^{m}, \mathbf{x}=\left(x_{1}, . ., x_{m}\right), \mathbf{y}=\left(y_{1}, . ., y_{m}\right)$,

$$
\begin{aligned}
& \mathbf{x} \leq \mathbf{y} \Leftrightarrow x_{i} \leq y_{i}, \text { for all } i=1, . ., m . \\
& \mathbf{x}<\mathbf{y} \Leftrightarrow x_{i}<y_{i}, \text { for all } i=1, . ., m .
\end{aligned}
$$

We follow Freixas and Zwicker's (2003) general definition of weighted (4,2)-rules.

Definition 25 An n-voter quaternary dichotomous weighted rule is specified by a system of weights $\mathbf{w}^{Y}=\left(w_{1}^{Y}, . ., w_{n}^{Y}\right), \mathbf{w}^{A}=\left(w_{1}^{A}, . ., w_{n}^{A}\right), \mathbf{w}^{H}=\left(w_{1}^{H}, . ., w_{n}^{H}\right), \mathbf{w}^{N}=$ $\left(w_{1}^{N}, . ., w_{n}^{N}\right)$ such that for all $i$

$$
w_{i}^{Y} \geq \max \left\{w_{i}^{A}, w_{i}^{H}, w_{i}^{N}\right\}
$$

and $\mathbf{w}^{A}, \mathbf{w}^{H}$ and $\mathbf{w}^{N}$ are linearly (pre)ordered (i.e. such that $\mathbf{w}^{X} \leq \mathbf{w}^{Z}$ or $\mathbf{w}^{Z} \leq \mathbf{w}^{X}$ for any $X, Z \in\{A, H, N\}$ ), and by a quota $Q>0$, so that a vote configuration $S \in 4^{\mathcal{N}}$ is winning if and only if

$$
\sum_{i \in S^{Y}} w_{i}^{Y}+\sum_{i \in S^{A}} w_{i}^{A}+\sum_{i \in S^{H}} w_{i}^{H}+\sum_{i \in S^{N}} w_{i}^{N}>Q
$$

Such a rule is denoted by $\mathcal{Q}\left(Q ; \mathbf{w}^{Y}, \mathbf{w}^{A}, \mathbf{w}^{H}, \mathbf{w}^{N}\right)$. 
This definition ${ }^{6}$ constrains the weights by (8) so as to make the rule consistent with the basic monotonicities assumed for all QVRs. Moreover, the ranking of the weights corresponds to the monotonicities. For instance, if $\mathbf{w}^{Y}>\mathbf{w}^{A}>\mathbf{w}^{H}>\mathbf{w}^{N}$ the rule belongs to the monotonic class whose monotonicities are $N \rightarrow H \rightarrow A \rightarrow Y$; while if $\mathbf{w}^{Y}>\mathbf{w}^{A}=\mathbf{w}^{H}>\mathbf{w}^{N}$ it belongs to the class where $N \rightarrow H \equiv A \rightarrow Y$, etc. In other words, all weighted rules belong to the linear classes introduced in Section 3. As we prove later, the class of weighted rules is rich enough to express any QVR as a 'dichotomous quaternary $k$-multiple weighted rule'. That is, as the intersection of $k$ weighted rules $\bigcap_{r=1}^{r=k} \mathcal{Q}\left(Q_{r} ; \mathbf{w}_{r}^{Y}, \mathbf{w}_{r}^{A}, \mathbf{w}_{r}^{H}, \mathbf{w}_{r}^{N}\right)$. More precisely, we have the following definition.

Definition 26 An n-voter dichotomous quaternary $k$-multiple weighted rule is specified by $k$ systems of weights $\mathbf{w}_{r}^{Y}=\left(w_{r 1}^{Y}, . ., w_{r n}^{Y}\right), \mathbf{w}_{r}^{A}=\left(w_{r 1}^{A}, . ., w_{r n}^{A}\right), \mathbf{w}_{r}^{H}=\left(w_{r 1}^{H}, . ., w_{r n}^{H}\right)$, $\mathbf{w}_{r}^{N}=\left(w_{r 1}^{N}, . ., w_{r n}^{N}\right)$ (where $\left.r=1, . ., k\right)$, and $k$ quotas $Q_{r}$, such that for each $r$

$$
\mathcal{Q}\left(Q_{r} ; \mathbf{w}_{r}^{Y}, \mathbf{w}_{r}^{A}, \mathbf{w}_{r}^{H}, \mathbf{w}_{r}^{N}\right)
$$

is a quaternary dichotomous weighted rule according to Def. 25, so that a vote configuration $S \in 4^{\mathcal{N}}$ is winning if and only if

$$
\sum_{i \in S^{Y}} w_{r i}^{Y}+\sum_{i \in S^{A}} w_{r i}^{A}+\sum_{i \in S^{H}} w_{r i}^{H}+\sum_{i \in S^{N}} w_{r i}^{N}>Q_{r}, \text { for all } r=1, . ., k .
$$

Now the point is the representability of all QVRs by means of weighted rules. Let us first address the representability of rules belonging to the linear classes.

For the case of the linear classes, given the fact that these classes correspond to the possible orders between weights consistent with the constraints in Definition 25, the result of Freixas and Zwicker (2003) applies straightforwardly. That is, their characterization theorem answers the question of the necessary and sufficient conditions for the representability of a linear QVR as a (single) weighted rule. But there is the question of the well-definedness of the notion of 'dimension' for general quaternary rules. The following result extends the proof of Taylor and Zwicker (1999) to the case of linear classes of monotonic rules, showing that the notion of dimension is sound for quaternary rules in the linear classes.

Theorem 27 Let $C$ be a linear class of monotonic rules, then for any rule $\mathcal{W} \in C$ there exists a dichotomous quaternary $k$-multiple weighted rule $\mathcal{Q}$ such that $\mathcal{W}=\mathcal{Q}$.

\footnotetext{
${ }^{6}$ In order to grant that a QVR consistent with Definition 4 results, two conditions should be added: first, to ensure 'full-support', $\sum_{i \in \mathcal{N}} w_{i}^{Y}>Q$, and, second, either to ensure 'null-support',

$$
\sum_{j \in S^{A}} w_{j}^{A}+\sum_{k \in S^{H}} w_{k}^{H}+\sum_{l \in S^{N}} w_{l}^{N} \leq Q
$$
}

for any $S$ such that $S^{Y}=\varnothing$, or a somewhat more complicated condition to ensure 'weak null-support'. 
Proof. We provide the proof for class $C 4$, whose monotonicities are

$$
N \rightarrow H \rightarrow A \rightarrow Y
$$

but the proof is entirely analogous for any linear class. With the notation introduced in Section 3, we have $N<_{C 4} H<_{C 4} A<_{C 4} Y$. Given a vote configuration $S=$ $\left(S^{Y}, S^{A}, S^{H}, S^{N}\right)$, for each $i \in \mathcal{N}$ and $X \in\{Y, A, H, N\}$, we denote $S^{-1}(i)=X$ if $i \in S^{X}$. Let $\mathcal{W}$ be a rule in $C 4$. The proof consists of constructing a weighted rule for each maximal (w.r.t. $\preceq_{C 4}$ ) losing vote configuration, and showing that $\mathcal{W}$ is the intersection of all of them. Let $L=\left(L^{Y}, L^{A}, L^{H}, L^{N}\right)$ be a maximal losing vote configuration in $\mathcal{W}$ relative to $\preceq_{C 4}$, that is, $L \in \operatorname{Maxl}_{C 4}(\mathcal{W})$. Consider the weighted rule $\mathcal{Q}\left(Q_{L} ; \mathbf{w}_{L}^{Y}, \mathbf{w}_{L}^{A}, \mathbf{w}_{L}^{H}, \mathbf{w}_{L}^{N}\right)$, such that $Q_{L}=1 / 2$, and its weight system is given by:

\begin{tabular}{c|c|c|c|c|}
\multicolumn{1}{c}{} & \multicolumn{1}{c}{$i \in L^{Y}$} & $i \in L^{A}$ & $i \in L^{H}$ & $i \in L^{N}$ \\
\cline { 2 - 5 }$w_{L i}^{Y}$ & 0 & 1 & 1 & 1 \\
\cline { 2 - 5 }$w_{L i}^{A}$ & 0 & 0 & 1 & 1 \\
$w_{L i}^{H}$ & 0 & 0 & 0 & 1 \\
\cline { 2 - 5 }$w_{L i}^{N}$ & 0 & 0 & 0 & 0 \\
\cline { 2 - 5 } & & &
\end{tabular}

That is to say:

$$
\begin{gathered}
w_{L i}^{Y}:=\left\{\begin{array}{l}
0, \text { if } i \in L^{Y} \\
1, \text { if } i \in L^{A} \cup L^{H} \cup L^{N}
\end{array}\right. \\
w_{L i}^{A}:=\left\{\begin{array}{l}
0, \text { if } i \in L^{Y} \cup L^{A} \\
1, \text { if } i \in L^{H} \cup L^{N}
\end{array}\right. \\
w_{L i}^{H}:=\left\{\begin{array}{l}
0, \text { if } i \in L^{Y} \cup L^{A} \cup L^{H} \\
1, \text { if } i \in L^{N}
\end{array}\right. \\
w_{L i}^{N}:=0, \text { for all } i \in \mathcal{N} .
\end{gathered}
$$

Obviously, $\mathbf{w}^{Y} \geq \mathbf{w}^{A} \geq \mathbf{w}^{H} \geq \mathbf{w}^{N}$, and $\operatorname{Maxl}_{C 4}\left(\mathcal{Q}\left(Q_{L} ; \mathbf{w}_{L}^{Y}, \mathbf{w}_{L}^{A}, \mathbf{w}_{L}^{H}, \mathbf{w}_{L}^{N}\right)\right)=\{L\}$ (to see this, note that the cells in the diagonal contain the weights to be added corresponding to vote configuration $L$, all of them 0 , and any transfer of votes from this configuration in the sense of the monotonicities in $C 4$ would yield a winning configuration). Then we have

$$
\begin{gathered}
S=\left(S^{Y}, S^{A}, S^{H}, S^{N}\right) \in \mathcal{W} \Leftrightarrow \forall L \in \operatorname{Maxl}_{C 4}(\mathcal{W}): S \npreceq_{C 4} L \\
\Leftrightarrow \forall L \in \operatorname{Maxl}_{C 4}(\mathcal{W}): \exists i \text { s.t. } L^{-1}(i)<_{C 4} S^{-1}(i) \\
\Leftrightarrow \forall L \in \operatorname{Maxl}_{C 4}(\mathcal{W}): \exists i \text { s.t. } w_{L i}^{S^{-1}(i)}=1 \\
\Leftrightarrow \forall L \in \operatorname{Maxl}_{C 4}(\mathcal{W}): \sum_{i \in \mathcal{N}} w_{L i}^{S^{-1}(i)} \geq 1>1 / 2 \\
\Leftrightarrow \forall L \in \operatorname{Maxl}_{C 4}(\mathcal{W}): S \in \mathcal{Q}\left(Q_{L} ; \mathbf{w}_{L}^{Y}, \mathbf{w}_{L}^{A}, \mathbf{w}_{L}^{H}, \mathbf{w}_{L}^{N}\right) .
\end{gathered}
$$


Therefore

$$
\mathcal{W}=\bigcap_{L \in \operatorname{Maxl}_{C 4}(\mathcal{W})} \mathcal{Q}\left(Q_{L} ; \mathbf{w}_{L}^{Y}, \mathbf{w}_{L}^{A}, \mathbf{w}_{L}^{H}, \mathbf{w}_{L}^{N}\right) .
$$

In other words, $\mathcal{W}$ is a quaternary $k$-multiple weighted rule with $k=\# \operatorname{Maxl}_{C 4}(\mathcal{W})$.

Remark: Observe that $\mathcal{Q}\left(Q_{L} ; \mathbf{w}_{L}^{Y}, \mathbf{w}_{L}^{A}, \mathbf{w}_{L}^{H}, \mathbf{w}_{L}^{N}\right)$ satisfies full support and null support, but, as is the case for binary rules (see Taylor and Zwicker, 1999), it is not proper in the sense of Def. 22.

Therefore there only remains the question of the representability of QVRs in the 'non linear' classes. In view of Theorem 27, it is enough to prove that any rule in any class that is not linear can be represented as the intersection of a finite set of linear rules. In fact, this can be derived as a consequence of Szpilrajn's (1930) extension theorem, given that any partial order can be extended to a linear order and is the intersection of all such extensions. Nevertheless, we provide a direct proof showing in addition that only two linear rules are needed.

Proposition 28 Any quaternary voting rule $\mathcal{W}$ either is linear or is the intersection of two linear rules.

Proof. Assume $\mathcal{W}$ is not linear. In view of Proposition 17, it is enough to see that, whatever the monotonic class $C$ belongs to, there exist two linear classes, $C_{1}$ and $C_{2}$ such that $C=C_{1} \vee C_{2}$. We prove that this is so for the six types of class corresponding to the six essentially different non linear configurations of monotonicities (as discussed in Section 3). Assume $\mathcal{W} \in C$. For the six possible cases for $C$ we give two linear classes whose join is $C$. We specify the classes in terms of their diagrams of monotonicities ${ }^{7}$.

$$
\begin{aligned}
& \text { 1. }\left[\begin{array}{cc}
Y & \\
Z \equiv X & V
\end{array}\right]=\left[\begin{array}{c}
Y \\
\uparrow \\
Z \underset{\equiv}{ } \underset{\uparrow}{\uparrow} \\
V
\end{array}\right] \cap\left[\begin{array}{c}
Y \\
\uparrow \\
V \\
\uparrow \\
Z \equiv X
\end{array}\right] . \\
& \text { 2. }\left[\begin{array}{cc}
Y \equiv Z \\
\nearrow & \nwarrow \\
X & V
\end{array}\right]=\left[\begin{array}{c}
Y \equiv Z \\
\uparrow \\
X \\
\uparrow \\
V
\end{array}\right] \cap\left[\begin{array}{c}
Y \equiv Z \\
\uparrow \\
V \\
\uparrow \\
X
\end{array}\right] \text {. }
\end{aligned}
$$

\footnotetext{
${ }^{7}$ In other terms, we show explicitly how for each class $C$, the associated partial order $\leq_{C}$ has Dushnik-Miller's (1941) dimension 2.
} 


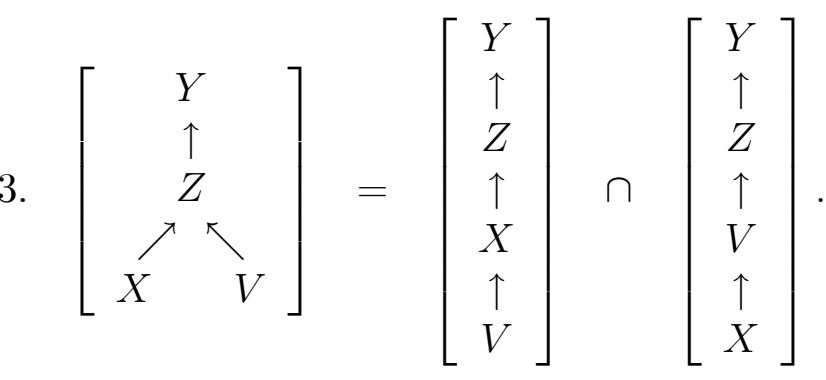

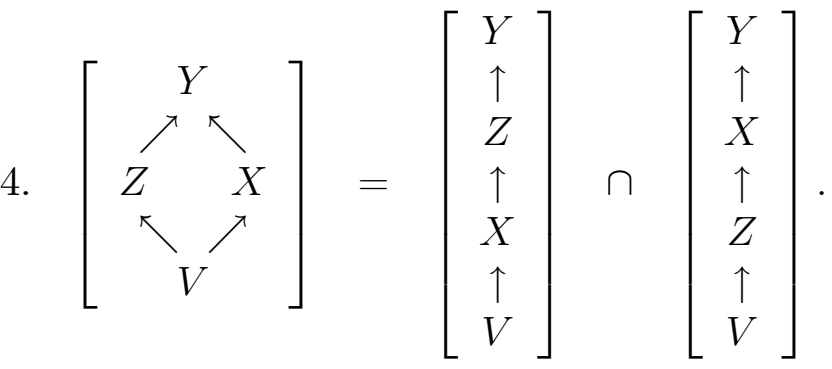

$5 .\left[\begin{array}{cc}\multicolumn{1}{c}{Y} & \\ Y & \nwarrow \\ Z & V \\ \uparrow & \\ X & \end{array}\right]=\left[\begin{array}{c}Y \\ \uparrow \\ Z \\ \uparrow \\ X \\ \uparrow \\ V\end{array}\right] \cap\left[\begin{array}{c}Y \\ \uparrow \\ V \\ \uparrow \\ Z \\ \uparrow \\ X\end{array}\right]$.

6. $\left[\begin{array}{ccc} & Y & \\ & Y \uparrow & \\ Z & V & X\end{array}\right]=\left[\begin{array}{c}Y \\ \uparrow \\ Z \\ \uparrow \\ V \\ \uparrow \\ X\end{array}\right] \cap\left[\begin{array}{c}Y \\ \uparrow \\ X \\ \uparrow \\ V \\ \uparrow \\ Z\end{array}\right]$.

It is easy to check that in all cases $\leq_{C}=\leq_{C_{1}} \cap \leq_{C_{2}}$.

The decompositions given in the proof are not unique. For instance, case 5 can alternatively be expressed in the following way:

$$
\left[\begin{array}{cc}
\multicolumn{2}{c}{Y} \\
\nearrow & \\
Z & V \\
\uparrow & \\
X &
\end{array}\right]=\left[\begin{array}{c}
Y \equiv V \\
\uparrow \\
Z \\
\uparrow \\
X
\end{array}\right] \cap\left[\begin{array}{c}
Y \equiv Z \equiv X \\
\uparrow \\
V
\end{array}\right]
$$

As a consequence we have the following 
Corollary 29 All quaternary voting rules can be represented as the intersection of a finite number of weighted QVRs. In particular, non linear $Q V R$ s can only be represented as the intersection of at least two weighted QVRs.

Proof. By Theorem 27, all linear rules are representable in this way. By Proposition 28, non linear QVRs can be represented as the intersection of two linear ones, but never as a single linear rule. This is because a weighted QVR is always linear.

Therefore the notion of dimension of a QVR as the minimal number of weighted QVRs by means of which it can be expressed ${ }^{8}$ is well defined for any QVR, and non linear QVRs have at least dimension two.

\section{Concluding remarks}

We have provided a model of quaternary voting rule that covers dichotomous rules admitting abstention and 'staying home' as legitimate and possibly differentiated options, and covers all real world dichotomous voting rules based on these four options and different types of 'quorum' that we know of. The model extends consistently simpler models such as binary and ternary voting rules. In particular, notions such as that of 'proper' rule and 'minimal winning' and 'maximal losing' vote configurations have been naturally extended. We have also extended the notion of weighted rule to this enlarged class of rules and the notion of dimension. We have also proved the representability of all QVR as the intersection of such weighted rules.

As main lines of further research, the following seem worth investigating. In contrast with binary dichotomous voting rules, actions do not follow trivially from preferences even when all voters have strict preferences when the QVR rule is not linear. The framework provided suggests a study of different classes of rules and rules to be found in parliaments from this point of view. The framework provided also suggests a systematic study of different anonymous majority rules in parliaments and other decision-making bodies, and perhaps founding reasonable proposals.

\section{References}

[1] Côrte-Real, P., Pereira, P.T., 2004. The voter who wasn't there: Referenda, representation and abstention. Social Choice and Welfare 22, 349-369.

[2] Dougherty, K. L., Edward, J., 2010. The Properties of Simple vs. Absolute Majority Rule: Cases where Absences and Abstentions are Important. Journal of Theoretical Politics 22 (forthcoming).

[3] Dushnik, B., Miller, E. W., 1941. Partially ordered sets, American Journal of Mathematics 63, 600-610.

\footnotetext{
${ }^{8}$ Equivalently the minimal $k$ for which it can be expressed as a $k$-multiple weighted QVR.
} 
[4] Felsenthal, D. S., Machover, M., 1997. Ternary Voting Games. International Journal of Game Theory 26, 335-351.

[5] Felsenthal, D. S., Machover, M., 1998. The Measurement of Voting Power: Theory and Practice, Problems and Paradoxes. Edward Elgar Publishers, London.

[6] Freixas, J., Zwicker, W., 2003. Weighted Voting, Abstention and Multiple Levels of Approval. Social Choice and Welfare 21, 399-431.

[7] Freixas, J., Zwicker, W., 2009. Anonymous yes-no voting with abstention and multiple levels of approval. Games and Economic Behavior 67, 428-444.

[8] Laruelle, A., Valenciano, F., 2008. Voting and Collective Decision-Making: Bargaining and Power. Cambridge University Press, Cambridge, New York.

[9] Maniquet, F., Morelli, M. 2008. Participation versus Approval Quorums, mimeo.

[10] Szpilrajn, E., 1930. Sur l'extension de l'ordre partiel. Fundamenta Mathematicae $16,1251-1256$.

[11] Taylor, A. D., Zwicker, W. S., 1992, A Characterization of Weighted Voting, Proceedings of the American Mathematical Society 115, 1089-1094.

[12] Taylor, A. D., Zwicker, W. S., 1993. Weighted Voting, Multicameral Representation and Power. Games and Economic Behavior 5, 170-181.

[13] Taylor A. D., Zwicker, W. S., 1999. Simple Games: Desirability Relations, Trading, Pseudoweightings. Princeton University Press.

[14] Vermeule, A., 2007. Absolute majority rules, British Journal of Political Science $37,643-658$.

[15] Zwicker, W., 2009. Anonymous voting rules with abstention: Weighted voting. In: Brams, S. J., Gehrlein, W. V., Roberts, F. S. (Eds.), The Mathematics of Preference, Choice, and Order: Essays in Honor of Peter C. Fishburn. Springer, Heidelberg, 239-258. 